\title{
Radiation Dosimetry of ${ }^{89} \mathrm{Zr}$-Labeled Chimeric Monoclonal Antibody U36 as Used for Immuno-PET in Head and Neck Cancer Patients
}

Pontus K.E. Börjesson ${ }^{1}$, Yvonne W.S. Jauw ${ }^{1}$, Remco de Bree ${ }^{1}$, Jan C. Roos ${ }^{2}$, Jonas A. Castelijns ${ }^{3}$, C. René Leemans ${ }^{1}$, Guus A.M.S. van Dongen ${ }^{1,2}$, and Ronald Boellaard ${ }^{2}$

${ }^{I}$ Department of Otolaryngology/Head and Neck Surgery, VU University Medical Center, Amsterdam, The Netherlands;

${ }^{2}$ Department of Nuclear Medicine and PET Research, VU University Medical Center, Amsterdam, The Netherlands; and

${ }^{3}$ Department of Radiology, VU University Medical Center, Amsterdam, The Netherlands

Immuno-PET is an appealing concept in the detection of tumors and planning of antibody-based therapy. For this purpose, the long-lived positron emitter ${ }^{89} \mathrm{Zr}$ (half-life, $78.4 \mathrm{~h}$ ) recently became available. The aim of the present first-in-humans ${ }^{89} \mathrm{Zr}$ immunoPET study was to assess safety, biodistribution, radiation dose, and quantification of the ${ }^{89} \mathrm{Zr}$-labeled chimeric monoclonal antibody (cmAb) U36 in patients with head and neck squamous cell carcinoma (HNSCC). In addition, the performance of immunoPET for detecting lymph node metastases was evaluated, as described previously. Methods: Twenty HNSCC patients, scheduled to undergo surgical tumor resection, received 75 MBq of ${ }^{89} \mathrm{Zr}$-cmAb U36 (10 mg). Immuno-PET scans were acquired at $1,24,72$, or $144 \mathrm{~h}$ after injection. The biodistribution of the radioimmunoconjugate was evaluated by ex vivo radioactivity measurement in blood and in biopsies from the surgical specimen obtained at $168 \mathrm{~h}$ after injection. Uptake levels and residence times in blood, tumors, and organs of interest were derived from quantitative immuno-PET studies, and absorbed doses were calculated using OLINDA/EXM 1.0. The red marrow dose was calculated using the residence time for blood. Results: ${ }^{89} \mathrm{Zr}$-cmAb U36 was well tolerated by all subjects. PET quantification of blood-pool activity in the left ventricle of the heart showed a good agreement with sampled blood activity (difference equals $0.2 \% \pm 16.9 \%$ [mean $\pm \mathrm{SD}$ ]) except for heavyweight patients $(>100 \mathrm{~kg})$. A good agreement was also found for the assessment of mAb uptake in primary tumors (mean deviation, $-8.4 \% \pm 34.5 \%)$. The mean absorbed red marrow dose was $0.07 \pm 0.02 \mathrm{mSv} / \mathrm{MBq}$ and $0.09 \pm 0.01 \mathrm{mSv} / \mathrm{MBq}$ in men and women, respectively. The normal organ with the highest absorbed dose was the liver (mean dose, $1.25 \pm 0.27 \mathrm{mSv} / \mathrm{MBq}$ in men and $1.35 \pm 0.21 \mathrm{mSv} / \mathrm{MBq}$ in women), thereafter followed by kidneys, thyroid, lungs, and spleen. The mean effective dose was $0.53 \pm 0.03 \mathrm{mSv} / \mathrm{MBq}$ in men and $0.66 \pm 0.03 \mathrm{mSv} / \mathrm{MBq}$ in women. Measured excretion via the urinary tract was less than $3 \%$ during the first $72 \mathrm{~h}$. Conclusion: ${ }^{89} \mathrm{Zr}$ immuno-PET can be safely used to quantitatively assess biodistribution, uptake, or-

Received May 6, 2009; revision accepted Jul. 20, 2009.

For correspondence or reprints contact: Ronald Boellaard, Department of Nuclear Medicine and PET Research, VU University Medical Center,

De Boelelaan 1117, 1081 HV Amsterdam, The Netherlands.

E-mail: r.boellaard@vumc.nl

COPYRIGHT @ 2009 by the Society of Nuclear Medicine, Inc. gan residence times, and radiation dose, justifying its further clinical exploitation in the detection of tumors and planning of $\mathrm{mAb}-$ based therapy.

Key Words: immuno-PET; molecular imaging; head and neck cancer patients; ${ }^{89} \mathrm{Zr}$-labeled monoclonal antibody; dosimetry

J Nucl Med 2009; 50:1828-1836

DOI: 10.2967/jnumed.109.065862

\section{M} onoclonal antibodies (mAbs) have been approved for use as diagnostics and therapeutics in a broad range of medical indications, but especially in oncology (1). Immuno-PET, the tracking and quantification of mAbs with PET in vivo, is an exciting novel option to improve diagnostic imaging and to guide mAb-based therapy and has been described previously (2-6).

To enable PET of mAbs, an appropriate positron emitterwith a half-life $\left(t_{1 / 2}\right)$ that is compatible with the time needed to achieve optimal tumor-to-nontumor ratios (typically 2-4 d for intact mAbs) — has to be securely coupled to the targeting molecule. ${ }^{124} \mathrm{I}\left(\mathrm{t}_{1 / 2}, 100.3 \mathrm{~h}\right)$ and ${ }^{89} \mathrm{Zr}\left(\mathrm{t}_{1 / 2}\right.$, $78.4 \mathrm{~h}$ ) are particularly suitable in combination with intact mAbs, because their long half-lives allow imaging at late time points for obtaining maximum information. Although the nonresidualizing positron emitter ${ }^{124} \mathrm{I}$ is particularly suitable for immuno-PET when used in combination with noninternalizing intact $\mathrm{mAbs}$, the residualizing positron emitter ${ }^{89} \mathrm{Zr}$ may be optimal in combination with internalizing intact $\mathrm{mAbs}$, because ${ }^{89} \mathrm{Zr}$ stays in the targeted cell (residualization) after intracellular catabolism of the radioimmunoconjugate (7). ${ }^{89} \mathrm{Zr}$ can also be used as a PET surrogate label for the prediction of the biodistribution and dosimetry of ${ }^{177} \mathrm{Lu}-\mathrm{mAb}$ and ${ }^{90} \mathrm{Y}-\mathrm{mAb}$ conjugates as used in radioimmunotherapy trials, although deviations have to be anticipated because of subtle differences in the metalchelate complexes used $(8,9)$. 
Although the first clinical immuno-PET studies with ${ }^{124}$ I-labeled mAbs were performed about $15 \mathrm{y}$ ago, technology for ${ }^{89} \mathrm{Zr}$ immuno-PET became available just recently (10). For this purpose, we developed the large-scale production of pure ${ }^{89} \mathrm{Zr}$ and a strategy for labeling mAbs with ${ }^{89} \mathrm{Zr}$ via a multistep synthesis, using a succinylated derivative of desferrioxamine $B$ (Df) as bifunctional chelate (10). Labeling technology is universal and, therefore, can be used for each individual $\mathrm{mAb}$ or other type of protein. In the meantime, several preclinical immuno-PET studies have been performed with ${ }^{89} \mathrm{Zr}$-labeled mAbs as a prelude to clinical trials, for example, with chimeric (mouse-human) mAb (cmAb) U36 (anti-CD44v6) (10), DN30 (anti-cMet) (11), G250 (anticarbonic anhydrase IX) (12), ibritumomab tiuxetan and rituximab (anti-CD20) (9), bevacizumab (antivascular endothelial growth factor) (13), cetuximab (antiepidermal growth factor receptor) $(8,14)$, and trastuzumab (antihuman epidermal growth factor receptor-2) (15).

In a first-in-humans ${ }^{89} \mathrm{Zr}$ immuno-PET clinical trial, we recently determined the diagnostic value of immuno-PET with anti-CD44v6 ${ }^{89} \mathrm{Zr}-\mathrm{cmAb} \mathrm{U} 36$ in patients with head and neck squamous cell carcinoma (HNSCC), who were at high risk of having neck lymph node metastases (2). Twenty HNSCC patients underwent ${ }^{89} \mathrm{Zr}$-cmAb U36 immuno-PET before surgery. Immuno-PET detected all primary tumors ( $n=17$ ) and lymph node metastases in 18 of 25 positive neck levels. For the detection of HNSCC lymph node metastases (and probably distant metastases), immunoPET with ${ }^{89} \mathrm{Zr}$-cmAb U36 performs at least as well as CT or MRI.

No radiation dose estimates have been previously described for ${ }^{89} \mathrm{Zr}$-cmAb U36 or other ${ }^{89} \mathrm{Zr}$-labeled mAbs. Therefore, the aim of the present study was to assess the safety, biodistribution, radiation dose, and potential for quantification of immuno-PET with ${ }^{89} \mathrm{Zr}-\mathrm{cmAb}$ U36 in HNSCC patients using data from the aforementioned clinical trial.

\section{MATERIALS AND METHODS}

\section{Patient Study}

A total of 20 patients ( 8 women and 12 men) with histologically proven HNSCC (Table 1), at high risk of having neck lymph node metastasis and, therefore, planned to undergo neck dissection with or without resection of the primary tumor, participated in this study. Other eligibility criteria have been described previously (2). Patients received cmAb U36 IgG radiolabeled with ${ }^{89} \mathrm{Zr}$ (74.9 \pm $0.6 \mathrm{MBq}$ ). The total administered cmAb U36 dose was $10 \mathrm{mg}$ for all patients. In previous studies, it had been demonstrated that biodistribution is not $\mathrm{mAb}$ dose-dependent within the range of $2-52 \mathrm{mg}(16,17)$. Surgery was performed 6-8 d after the administration of radiolabeled $\mathrm{cmAb} \mathrm{U} 36$.

\section{Safety}

Before and up to $6 \mathrm{wk}$ after the administration of radiolabeled $\mathrm{cmAb} \mathrm{U} 36$, routine laboratory analyses were performed, including hemoglobin, hematocrit, mean corpuscular volume, red blood cell count, white blood cell count (including automated differential), platelet count, sodium, potassium, calcium, chloride, creatinine, urea, uric acid, alanine aminotransferase, aspartate aminotransferase, alkaline phosphatase, $\gamma$-glutamyl transferase, albumin, glucose, bilirubin, thyroid-stimulating hormone, and urine sediment. Vital signs including pulse rate, blood pressure, temperature, and respiratory rate were recorded before and up to $3 \mathrm{~h}$ after injection. On the basis of previous studies with anti-CD44v6 mAbs, cmAb U36 included, no adverse effects were expected (17). Dose rates $(\mu \mathrm{Sv} / \mathrm{h})$ were measured at 1,24 , and $72 \mathrm{~h}$ after injection at a distance of $100 \mathrm{~cm}$ with a $\gamma$-radiation dose rate counter (Berthold

\section{TABLE 1. Patient and Tumor Characteristics}

\begin{tabular}{|c|c|c|c|c|c|c|}
\hline Patient no. & Sex & Age $(y)$ & Weight $(\mathrm{kg})$ & Primary tumor & cTNM & pTNM \\
\hline 1 & $\mathrm{~F}$ & 57 & 68 & Oral cavity, tongue, right & T2NOMO & T2N1M0 \\
\hline 2 & $\mathrm{M}$ & 57 & 82 & Oropharynx, base of tongue, right & TXN2aM0 & T1N2bM0 \\
\hline 3 & $\mathrm{~F}$ & 72 & 71 & Oropharynx, tonsil, right & T2N2bM0 & T2N2bMO \\
\hline 4 & $\mathrm{~F}$ & 53 & 81 & Oropharynx, tonsil, right & T3N0MO & T3N2bM0 \\
\hline 5 & M & 63 & 78 & Oropharynx, tonsil, right & T4NOMO & T4N2bM0 \\
\hline 6 & $\mathrm{~F}$ & 58 & 62 & Oral cavity, floor of mouth, left & T4NOMO & T4N2bMO \\
\hline 7 & M & 54 & 67 & Oral cavity, tongue, right & T3NOMO & T3N2bM0 \\
\hline 8 & $\mathrm{M}$ & 55 & 72 & Oropharynx, tonsil, left & T4N2aM0 & T4N2bM0 \\
\hline 9 & $\mathrm{~F}$ & 54 & 82 & Hypopharynx, piriform sinus, left & T4N2bM0 & T4N2bM0 \\
\hline 10 & $\mathrm{~F}$ & 65 & 67 & Oral cavity, base of tongue, right & T2NOMO & T2NOMO \\
\hline 11 & $\mathrm{M}$ & 53 & 90 & Larynx, glottic & T4N0MO & T4N0M0 \\
\hline 12 & M & 59 & 75 & Larynx, supraglottic & T4NOMO & T4NOMO \\
\hline 13 & $\mathrm{~F}$ & 49 & 49 & Residual disease of T2N0 tonsil carcinoma, left & NA & NA \\
\hline 14 & M & 58 & 100 & Oropharynx, base of tongue, right & T2N2bM0 & T2N2bMO \\
\hline 15 & $\mathrm{M}$ & 48 & 115 & Oropharynx, tonsil, right & T3NOMO & T3N1M0 \\
\hline 16 & $\mathrm{~F}$ & 63 & 67 & Oropharynx, tonsil, left & T3N2bM0 & T3N2cM0 \\
\hline 17 & $\mathrm{M}$ & 53 & 104 & Oropharynx, tonsil, right & T2N3M0 & T2N3M0 \\
\hline 19 & $\mathrm{M}$ & 71 & 87 & Oropharynx, soft palate, right & T3N2cM0 & T2N2cMO \\
\hline 20 & $\mathrm{M}$ & 60 & 58 & Larynx, supraglottic, recurrence & NA & NA \\
\hline
\end{tabular}

For patient 18 , not enough data were available for adequate dosimetric analysis. NA $=$ not applicable. 
LB 1230; EG\&G). In addition, human anti-cmAb U36 and antiDf-cmAb U36 antibody responses were assessed (2).

\section{mAb U36}

The selection and production of mouse mAb U36 and its chimeric IgG1 derivative cmAb U36 have been described previously (17). mAb U36 binds to the v6 region of CD44 (CD44v6). Expression of CD44v6 was found to be abundant and homogeneous in $96 \%$ of all primary HNSCC and HNSCC lymph node metastases (18). In normal tissues, expression has been found in epithelial tissues such as skin, breast and prostate myoepithelium, and bronchial epithelium (19). CD44v6 has been suggested to be involved in tumor formation, tumor cell invasion, metastasis formation, and cancer cell stemness $(20,21)$.

\section{Synthesis of ${ }^{89} \mathrm{Zr}-\mathrm{cm} \mathrm{Ab}$ U36}

The synthesis and purification of ${ }^{89} \mathrm{Zr}$, its coupling to $\mathrm{cmAb}$ U36 via the chelate Df (Desferal; Novartis Pharma AG), and the procedures and results of quality tests have been described previously (2). ${ }^{89} \mathrm{Zr}$ emits positrons with a main energy of 897 $\mathrm{keV}$ and an abundance of $22.7 \%$. In addition, nonprompt $909-\mathrm{keV}$ photons are emitted at an abundance of $99.9 \%$.

\section{Pharmacokinetics}

Serial blood samples were taken from a peripheral vein of the arm opposite the infusion site for the determination of activity at the following time points: 5, 10, and $30 \mathrm{~min}$ and 1, 2, 4, 16, 21, 72, and $168 \mathrm{~h}$ after the completion of infusion. Urine was collected at intervals of $0-24,24-48$, and 48-72 $\mathrm{h}$ after injection to determine renal excretion of ${ }^{89} \mathrm{Zr}$. Aliquots of blood, plasma, and urine samples were measured for ${ }^{89} \mathrm{Zr}$ activity in an isotope well-counter (1470 Wizard; Wallac), compared with an aliquot retained from the conjugate preparation, and corrected for decay. Blood activity was expressed as the percentage of the injected dose per kilogram.

\section{PET Acquisition}

PET scans were obtained at $1 \mathrm{~h}$ (all patients except 4 and 16), $24 \mathrm{~h}$ (patients 1-6), $72 \mathrm{~h}$ (all patients), or $144 \mathrm{~h}$ (all patients except 1,2 , and 13) after the intravenous injection of ${ }^{89} \mathrm{Zr}-\mathrm{cmAb} \mathrm{U} 36$, using a dedicated full-ring PET scanner (ECAT EXACT HR+; CTI/Siemens) as described before (2). Before this study, the PET scanner was calibrated using a standard cylindric calibration phantom filled with a ${ }^{89} \mathrm{Zr}$ solution $\left(5 \mathrm{kBq} / \mathrm{cm}^{3}\right)$. This calibration was performed to verify the quantitative accuracy of the scanner in the presence of $909-\mathrm{keV} \gamma$-photons emitted by ${ }^{89} \mathrm{Zr}$ and the impact of emission spillover into the transmission scans. This procedure indicated that activity concentrations measured with the HR+ were accurate within 5\%. During image reconstruction, all scans were normalized and corrected for randoms, scatter, attenuation, and decay. Reconstructions were performed using an attenuationand normalization-weighted ordered-subset expectation maximization (OSEM) algorithm (ECAT software, version 7.2; CTI/ Siemens) with 2 iterations and 16 subsets, followed by postsmoothing of the reconstructed image using a gaussian filter of $5 \mathrm{~mm}$ in full width at half maximum . Because of the low amount of radioactivity administered to the patients (for radiation exposure reasons), images with attenuation correction showed high noise levels. Therefore, OSEM reconstructions without attenuation (and thus scatter) correction were performed as well and used for visual interpretation only.
Validation of Quantitative ${ }^{89} \mathrm{Zr}$ Immuno-PET

OSEM reconstructions with attenuation correction of the immuno-PET scans were used to investigate the accuracy of quantification ${ }^{89} \mathrm{Zr}$-cmAb U36 PET in vivo. To this end, three 1.5$\mathrm{cm}$-diameter regions of interest (ROIs) were defined within the left ventricle of the heart in 3 subsequent image planes using an axial view of the PET images. The average activity concentration within these ROIs, representing PET-assessed blood-pool activity concentration, was directly compared with the activity concentration measured in manual blood samples (sampled blood activity) using a calibrated well-counter to validate the quantitative accuracy of ${ }^{89} \mathrm{Zr}$ PET in vivo.

In addition, the uptake of the radioimmunoconjugate in tumors (percentage injected dose $[\% \mathrm{ID}] / \mathrm{cm}^{3}$ ), as assessed from scans acquired at $144 \mathrm{~h}$ after injection, was compared with the uptake (\% ID/g) derived from tumor biopsies collected at $168 \mathrm{~h}$ after injection. $\% \mathrm{ID} / \mathrm{cm}^{3}$, as derived from the PET scans, was converted to $\% \mathrm{ID} / \mathrm{g}$ using a soft-tissue density factor of $1.04 \mathrm{~g} / \mathrm{cm}^{3}$.

\section{Volume-of-Interest (VOI) Definition}

The uptake in different organs was determined using manually defined ROIs and the Clinical Application Programming Package (CAPP; provided with the ECAT software). ${ }^{89} \mathrm{Zr}$ uptake was determined in the following organs: lung, liver, spleen, kidney, heart, and, if visible, thyroid. In addition, a semiautomatic VOI was defined over the tumor using in-house-developed software tools (22).

Details of ROI/VOI Definition. As most organs could be visually best identified on the 1-h-after-injection emission scans, most organ 3-dimensional (3D) VOIs were defined manually using this early uptake scan. However, VOIs for the thyroid and tumor were defined on the last scan, because their delineation was optimal at this last time point. Finally, lung regions were defined on reconstructed transmission scans, using the semiautomatic isocontour tool within the CAPP software. All 3D VOIs were defined using a coronal view of the PET images.

A 3D VOI was generated from multiple 2-dimensional ROIs by grouping these ROIs into the VOI. The ROIs on the first and last slices were not used for the VOIs, because these were prone to partial-volume effects (spill-in and spill-out) or sampling errors. The VOIs defined on the 1-h-after-injection scan were saved and imported to the other time frames, for which the organs were not as clearly visible. To allow for projection of VOIs onto scans obtained at other intervals, we scanned patients in the same (patient and bed) position during subsequent studies, using rigid head immobilization devices (e.g., radiotherapy mask) and belts around the patient. To ensure correct positioning of the VOIs, the elevation of the bed was registered for all scans and in the case of differences in patient or bed position, scans were aligned using an in-house-developed software program. Furthermore, in the case of remaining dislocation of VOIs, all VOIs were relocated in 1 step to keep the internal relations intact. For relocation, the alignment of lung VOIs and the contour of the head and neck were verified using reconstructed transmission scans (for each subsequent study) as well. However, if still necessary, VOIs were ungrouped and individual VOIs were relocated to improve the position. After this VOI positioning procedure, all VOIs were saved and projected onto the emission scans. The mean uptake (in $\mathrm{Bq} / \mathrm{cm}^{3}$ ) was then derived using these final VOIs.

The tumor was defined on the 144-h-after-injection scan using a tool that semiautomatically defined a 3D 50\% (to maximum) 
isocontour around the tumor. Then the mean uptake (in $\mathrm{Bq} / \mathrm{cm}^{3}$ ) was calculated. The mean tumor uptake was determined for the latest acquired PET study only, as correct repositioning of the tumor VOI onto earlier scans could not be reliably visually verified because of the lack of signal from the tumor.

All VOIs set on organs and tumors were evaluated by 2 experienced nuclear physicians.

\section{Internal Radiation Dosimetry}

The internal radiation dosimetry for the adult human was evaluated through the normalized cumulated activities for each patient provided as input to the OLINDA/EXM 1.0 code (23).

Residence times were calculated for liver, kidneys, lungs, spleen, and the remainder of the body, entering the percentage of the injected dose at each time point for each patient in OLINDA/EXM 1.0 and fitting these data using a monoexponential function. The residence times for the thyroid were calculated using trapezoidal integration. The remaining area under the curve from the end of data collection until infinity was determined by considering physical decay only. The residence time of the remainder of the body was defined as 113 minus the sum of residence times of source organs. A total residence time for the entire body of $113 \mathrm{~h}$ is obtained in the case of no biologic clearance. The red marrow dose was estimated using sampled blood clearance data $(24,25)$. The red marrow concentration was assumed to be $30 \%$ of the blood activity concentration (conversion factor, 0.3). In addition, the dose from the remainder of the body onto red marrow was considered as well.

\section{RESULTS}

Unless otherwise specified, all ${ }^{89} \mathrm{Zr}$ activities are decaycorrected to time of injection.

For 1 patient (patient 18) imaged, not enough data were available for adequate dosimetric analysis. Regarding the evaluation of ${ }^{89} \mathrm{Zr}$ immuno-PET to quantify tumor uptake, 4 patients (patients 1, 2, 13, and 18) were excluded from analysis because of the lack of a scan at $144 \mathrm{~h}$ after injection. Another patient (patient 6) was excluded because of delayed surgery $2 \mathrm{wk}$ after injection.

\section{Safety}

${ }^{89} \mathrm{Zr}-\mathrm{cmAb}$ U36 was found to be safe and well tolerated in all subjects. Neither adverse reactions nor significant changes in earlier mentioned blood and urine parameters that could be related to the study drug were observed. The mean radiation dose rates measured at 1,24 , and $72 \mathrm{~h}$ after injection at a distance of $100 \mathrm{~cm}$ were $7.0 \pm 0.3,5.7 \pm 0.3$, and $3.8 \pm 0.2 \mu \mathrm{Sv} / \mathrm{h}$, respectively. A human antichimeric antibody (HACA) response developed in patients 9 and 10, and elevated titers were found at 1 and $6 \mathrm{wk}$ after injection, irrespective of whether cmAb U36 IgG or ${ }^{89} \mathrm{Zr}-\mathrm{N}$-sucDf$\mathrm{cmAb}$ U36 was used in the enzyme-linked immunosorbent assay. These data indicate that the response was directed to the protein part of the conjugate and not to the $N$-sucDf chelate attached to the $\mathrm{cmAb}$.

\section{Biodistribution}

Images of a representative male patient between 1 and $144 \mathrm{~h}$ after injection of ${ }^{89} \mathrm{Zr}-\mathrm{cmAb} \mathrm{U} 36$ are presented in Figure 1. Whole-body images obtained directly after the administration of ${ }^{89} \mathrm{Zr}-\mathrm{cmAb}$ U36 showed mainly bloodpool activity, with delineation of the heart, lungs, liver, kidneys, spleen, and nose. Uptake of radioactivity for most organs decreased over time, whereas increased uptake was seen only at tumor sites and in the thyroid of some of the patients (patients 1, 6, 7, 10, and 11). The estimated uptake of ${ }^{89} \mathrm{Zr}-\mathrm{cmAb}$ U36 in single organs is presented in Table 2. The visual quality of the immuno-PET images varied between different patients. Figure 2 illustrates the difference
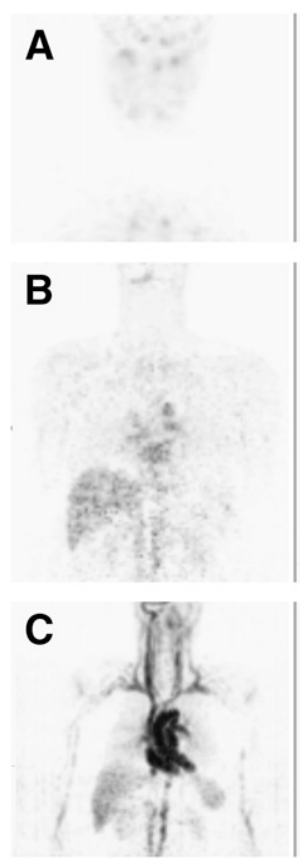
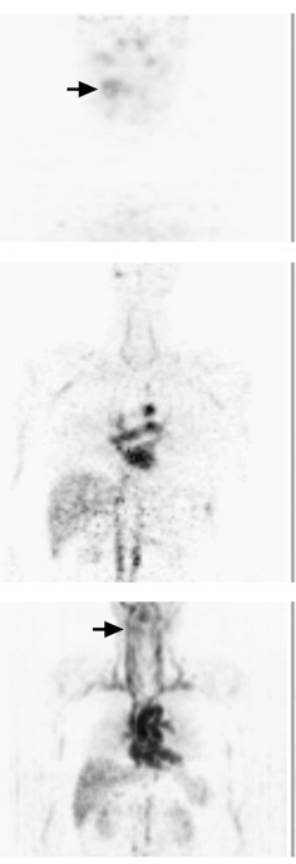
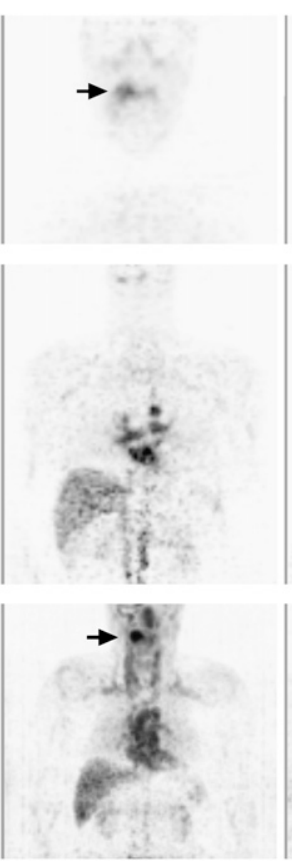

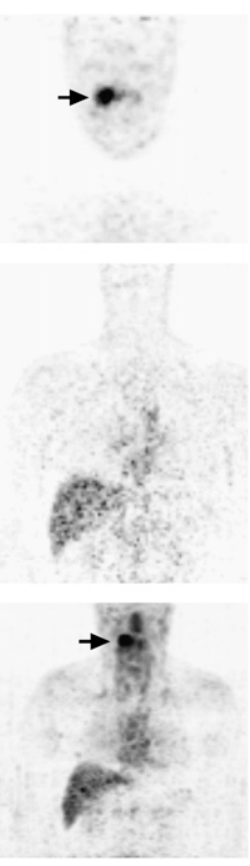

FIGURE 1. Representative coronal images of male patient with oropharyngeal tumor (indicated by arrows), arranged (left to right) from 1, 24, 72, and $144 \mathrm{~h}$ after injection. (A) Increased uptake in time of ${ }^{89} \mathrm{Zr}$-cmAb U36 in tumor (indicated by arrows), (B) Circulating ${ }^{89} \mathrm{Zr}$ cmAb U36 in heart and uptake in organs. (C) Maximum-intensity projections of same patient as shown in $A$ and B. Gray scale settings were set for each image independently, for clarity. 
TABLE 2. Uptake of ${ }^{89} \mathrm{Zr}-\mathrm{cmAb}$ U36 in Single Organs

\begin{tabular}{|c|c|c|c|c|c|c|c|c|}
\hline \multirow[b]{2}{*}{ Organ } & \multicolumn{4}{|c|}{ Time in men (h) } & \multicolumn{4}{|c|}{ Time in women (h) } \\
\hline & 0 & 24 & 72 & 144 & 0 & 24 & 72 & 144 \\
\hline Kidneys & $1.21 \pm 0.23$ & $1.36 \pm 0.14$ & $0.70 \pm 0.16$ & $0.26 \pm 0.09$ & $1.78 \pm 0.34$ & $1.69 \pm 0.44$ & $0.95 \pm 0.27$ & $0.37 \pm 0.07$ \\
\hline Liver & $12.23 \pm 2.12$ & $11.98 \pm 0.78$ & $6.18 \pm 1.44$ & $2.83 \pm 0.79$ & $11.50 \pm 2.45$ & $7.98 \pm 1.02$ & $4.94 \pm 0.95$ & $2.36 \pm 0.52$ \\
\hline Lungs & $4.51 \pm 3.58$ & $5.86 \pm 1.30$ & $1.84 \pm 1.20$ & $0.69 \pm 0.47$ & $5.91 \pm 1.94$ & $3.86 \pm 1.52$ & $2.52 \pm 0.75$ & $1.16 \pm 0.29$ \\
\hline Spleen & $0.99 \pm 0.27$ & $0.75 \pm 0.12$ & $0.29 \pm 0.10$ & $0.10 \pm 0.04$ & $1.02 \pm 0.36$ & $0.72 \pm 0.09$ & $0.27 \pm 0.12$ & $0.10 \pm 0.05$ \\
\hline Thyroid & $0.08 \pm 0.02$ & 0.11 & $0.07 \pm 0.04$ & $0.05 \pm 0.02$ & $0.06 \pm 0.03$ & $0.06 \pm 0.04$ & $0.10 \pm 0.03$ & $0.05 \pm 0.03$ \\
\hline
\end{tabular}

in visual quality between an average-weight patient $(58 \mathrm{~kg})$ and an obese patient $(104 \mathrm{~kg})$.

\section{PET-Derived Blood-Pool Activity Versus Sampled Blood}

Variation in visual image quality also affected the quantitative analysis of the ${ }^{89} \mathrm{Zr}-\mathrm{cmAb}$ U36 immuno-PET images in a proportion of patients, as shown in Figure 3. This plot depicts the percentage under- and overestimation of the blood activity as assessed by immuno-PET. To this end, immuno-PET-derived activity in the left ventricle of the heart was compared with activity in sampled blood. Patients with a weight lower than $100 \mathrm{~kg}$ showed, in general, a good agreement with manually sampled data (difference equals $0.2 \% \pm 16.9 \%$ [mean $\pm \mathrm{SD}$ ]). Patients with a weight more than $100 \mathrm{~kg}$ showed an underestimation of activity by immuno-PET, especially for later images. Figure 4 shows blood kinetics, assessed either by immuno-PET or by blood sampling, for the whole group of evaluable patients $(n=19)$. For the group of patients as a whole, no statistically significant $(P=0.503)$ differences were observed between the 2 methods used for assessment of activity. In addition, the small variances suggest a consistency of the pharmacokinetics in these patients, even for those in whom a HACA response developed. Therefore, we decided to use data on all these patients for radiation dose estimations.

A
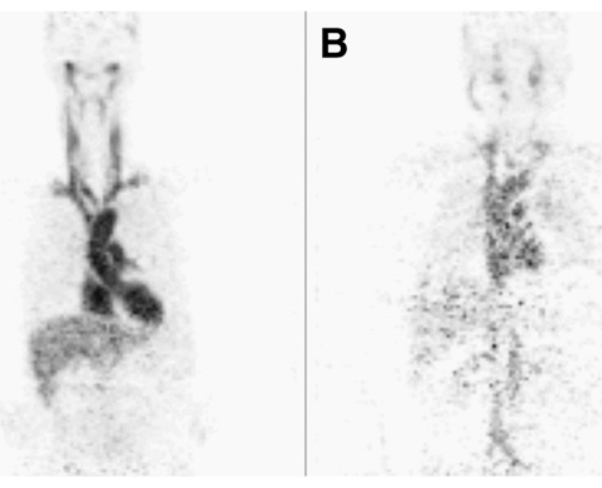

FIGURE 2. Difference in visual quality of coronal PET images obtained at $1 \mathrm{~h}$ after injection of ${ }^{89} \mathrm{Zr}$-cmAb U36 between average-weight patient $(58 \mathrm{~kg})(\mathrm{A})$ and obese patient (104 kg) (B).

\section{Quantification of mAb Uptake in Tumors}

Antibody uptake was assessed on 144-h-after-injection scans for all primary tumors and compared with uptake data from biopsies obtained at $168 \mathrm{~h}$ after injection. Comparison of \% ID/g derived from biopsy and PET data showed a good agreement with slightly lower values for PET (mean deviation, $-8.4 \% \pm 34.5 \%$ ). The mean tumor uptake at $168 \mathrm{~h}$ after injection as assessed by biopsies appeared to be $0.019 \pm$ $0.010 \% \mathrm{ID} / \mathrm{g}$ (range, $0.006-0.038 \% \mathrm{ID} / \mathrm{g}$ ).

\section{Red Marrow Doses}

The doses absorbed in the red marrow were estimated in 19 patients, as presented in Table 3. The mean red marrow dose was $0.07 \pm 0.02 \mathrm{mSv} / \mathrm{MBq}$ in men and $0.09 \pm 0.01$ $\mathrm{mSv} / \mathrm{MBq}$ in women.

\section{Absorbed Doses in Source Organs and Whole Body}

Residence times of the source organs were entered into the OLINDA/EXM 1.0 program. The results of the OLINDA/ EXM 1.0 analysis are listed in Table 4. The effective dose for each patient was calculated using the available organ dosimetry data of the source organs. Excretion of ${ }^{89} \mathrm{Zr}$ via the urinary pathway was $2.59 \pm 1.89 \%$ ID during the first $72 \mathrm{~h}$ after injection. As there was also little activity seen in the intestines, most loss of radioactivity was due to physical

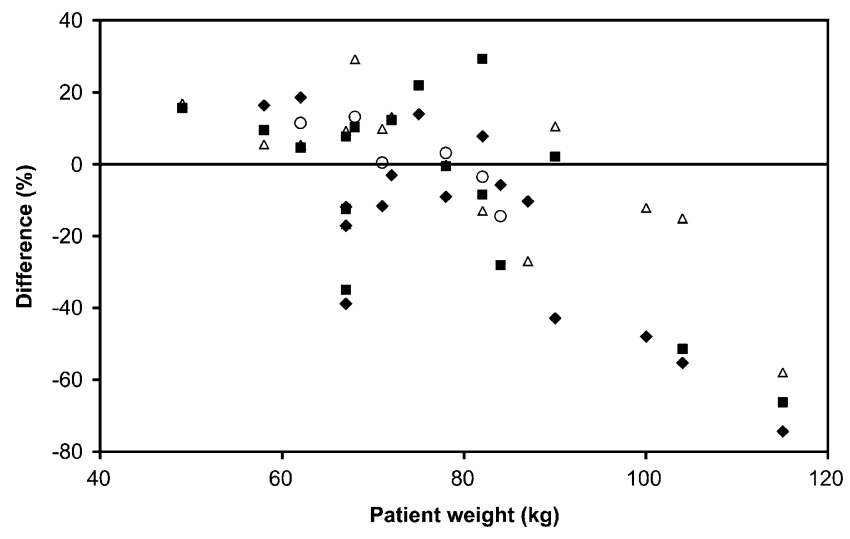

FIGURE 3. Difference between PET-assessed blood-pool activity and sampled blood activity of $89 \mathrm{Zr}-\mathrm{cmAb}$ U36 as function of patient weight. Difference in activity was assessed at $1(\triangle), 24(\bigcirc), 72(\bullet)$, and $144 \mathrm{~h}(\bullet)$ after injection. 


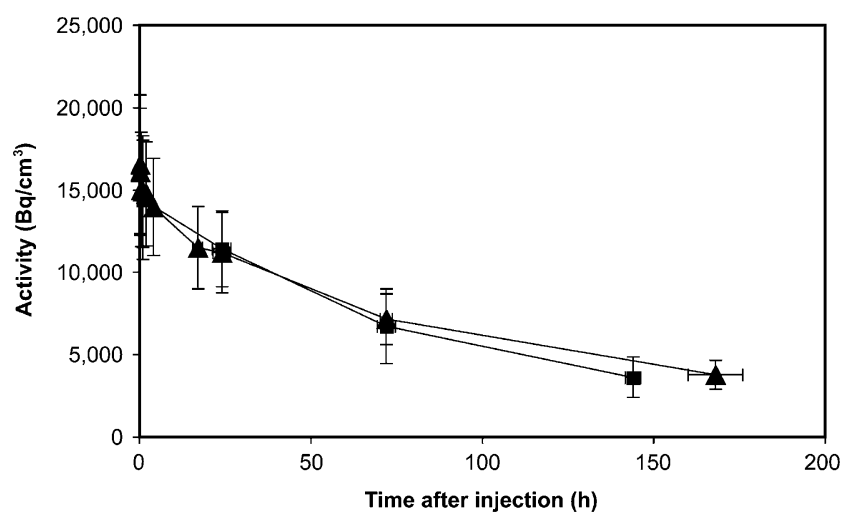

FIGURE 4. Mean ${ }^{89} \mathrm{Zr}$-cmAb U36 activity in blood $\left(\mathrm{Bq} / \mathrm{cm}^{3}\right)$ of study population: PET-assessed blood-pool activity within left ventricle of heart ( $\boldsymbol{\square})$ and sampled blood-pool activity $(\mathbf{\Delta})$.

decay. The mean effective dose for the whole body was $0.53 \pm 0.03 \mathrm{mSv} / \mathrm{MBq}$ in men and $0.66 \pm 0.03 \mathrm{mSv} / \mathrm{MBq}$ in women. As an alternative, the effective dose was also calculated by considering the body to be a homogeneous mass, that is, by ignoring organ doses and assuming a residence time of $113 \mathrm{~h}$ for the entire patient. This simplified procedure showed only a minor decrease of the estimated effective dose: $0.44 \pm 0.002 \mathrm{mSv} / \mathrm{MBq}$ in men and $0.54 \pm$ $0.002 \mathrm{mSv} / \mathrm{MBq}$ in women. The difference in estimated effective dose between men and women (with vs. without, ignoring the biodistribution) suggests that this difference

\section{TABLE 3. Absorbed Doses in Red Marrow}

\begin{tabular}{cc} 
Patient no. & Red marrow dose $(\mathrm{mSv} / \mathrm{MBq})$ \\
Men & 0.07 \\
2 & 0.07 \\
5 & 0.06 \\
7 & 0.06 \\
8 & 0.08 \\
11 & 0.07 \\
12 & 0.09 \\
14 & 0.11 \\
15 & 0.09 \\
17 & 0.07 \\
19 & 0.05 \\
20 & $0.07 \pm 0.02$ \\
Mean \pm SD & \\
Women & 0.09 \\
1 & 0.10 \\
3 & 0.11 \\
4 & 0.09 \\
6 & 0.11 \\
9 & 0.09 \\
10 & 0.07 \\
13 & 0.09 \\
16 & $0.09 \pm 0.01$ \\
Mean \pm SD & \\
& \\
${ }^{*}$ Conversion factor $=0.3$. & \\
\hline
\end{tabular}

might be explained by use of different models (men vs. women) within OLINDA/EXM 1.0.

The normal organs with the highest absorbed dose were the liver (mean dose in men, $1.25 \pm 0.27 \mathrm{mSv} / \mathrm{MBq}$; in women, $1.35 \pm 0.21 \mathrm{mSv} / \mathrm{MBq}$ ) and kidneys (mean dose in men, $0.82 \pm 0.15 \mathrm{mSv} / \mathrm{MBq}$; in women, $1.18 \pm 0.26 \mathrm{mSv} /$ $\mathrm{MBq})$.

\section{DISCUSSION}

The purpose of this study was to assess the safety and to evaluate the biodistribution, radiation dose, and potential for quantification of immuno-PET with ${ }^{89} \mathrm{Zr}$-labeled $\mathrm{cmAb}$ U36 in HNSCC patients. In this study, the tracer was found to be safe and well tolerated. No adverse events occurred. A HACA response was seen only in 2 patients, whereas none of the antibody responses was directed to the chelate. In all normal organs, the uptake of radioactivity decreased in time. Only in the tumor and in a few patients in the thyroid, uptake increased in time, suggesting specific uptake of ${ }^{89} \mathrm{Zr}-\mathrm{cmAb}$ U36. Such variable and sometimes high thyroid uptake was previously observed in HNSCC patients who had been injected with ${ }^{99 \mathrm{~m}} \mathrm{Tc}-\mathrm{cmAb} \mathrm{U} 36$ (16). This result might indicate that in some individuals CD44v6 is expressed in the thyroid.

Furthermore, the advantage of the more detailed images obtained with ${ }^{89} \mathrm{Zr}$ immuno-PET is the possibility of noninvasive quantification. In most of the images, the visual quality was acceptable for defining ROIs; however, there was a variation between images of different patients, and in some images the delineation of organs and tumor was suboptimal. Nevertheless, quantification results seem plausible. The ${ }^{89} \mathrm{Zr}$ calibration procedure indicated that the quantitative accuracy of the scanner was not compromised by the presence of $909-\mathrm{keV} \gamma$-photons emitted from ${ }^{89} \mathrm{Zr}$ and due to emission spillover into the transmission scans. In fact, the procedure was repeated for activity concentrations of about $17 \mathrm{kBq} / \mathrm{mL}(111 \mathrm{MBq}$ in a phantom of 6,283 $\mathrm{mL}$ ), showing that quantitative accuracy was not affected at higher counting rates as well. Although the $909-\mathrm{keV}$ photons could have resulted in increased dead time and randoms fraction, the ${ }^{89} \mathrm{Zr}$ activity in the field of view is much lower than usually applied (e.g., for ${ }^{18} \mathrm{~F}$-FDG studies, up to $370 \mathrm{MBq}$ of ${ }^{18} \mathrm{~F}-\mathrm{FDG}$ is applied for patients and about $70 \mathrm{MBq}$ is applied in a 6-L phantom for calibrations). Moreover, the $511-\mathrm{keV}$ photon flux from ${ }^{89} \mathrm{Zr}$ is much lower than that seen in ${ }^{18} \mathrm{~F}-\mathrm{FDG}$ studies because of lower positron emission abundance. Another effect that could hamper quantification might be emission spillover into the transmission scan. However, emission spillover into the transmission scan is minimized in 2 ways. First, transmission scans are based on coincidence counting of the 511$\mathrm{keV}$ photons emitted by the ${ }^{68} \mathrm{Ge}$ transmission rod sources, thereby reducing the detection of noncoincident emitted photons (although these still result in increased randoms fraction). Second, during transmission scanning a rod 
TABLE 4. Absorbed Organ Doses

\begin{tabular}{|c|c|c|c|c|c|c|c|}
\hline \multirow[b]{2}{*}{ Patient no. } & \multicolumn{6}{|c|}{ Organ dose (mSv/MBq) } & \multirow{2}{*}{$\begin{array}{c}\text { Effective dose } \\
(\mathrm{mSv} / \mathrm{MBq}) \text {, organ dosimetry }\end{array}$} \\
\hline & Kidneys & Liver & Lungs & Spleen & Thyroid & Total body & \\
\hline \multicolumn{8}{|l|}{ Men } \\
\hline 2 & 1.01 & 1.60 & 0.93 & 0.74 & 1.28 & 0.45 & 0.60 \\
\hline 5 & 0.85 & 1.47 & 0.83 & 0.66 & NV & 0.45 & 0.54 \\
\hline 7 & 0.86 & 1.20 & 0.68 & 0.74 & 0.70 & 0.44 & 0.53 \\
\hline 8 & 0.84 & 1.17 & 1.01 & 0.71 & NV & 0.44 & 0.55 \\
\hline 11 & 0.96 & 1.48 & 0.54 & 0.81 & 1.40 & 0.45 & 0.56 \\
\hline 12 & 1.01 & 1.55 & 0.53 & 0.82 & NV & 0.45 & 0.52 \\
\hline 14 & 0.73 & 1.01 & 0.44 & 0.58 & 0.86 & 0.44 & 0.52 \\
\hline 15 & 0.69 & 1.23 & 0.50 & 0.57 & 0.70 & 0.44 & 0.52 \\
\hline 17 & 0.58 & 0.69 & 0.42 & 0.47 & 0.72 & 0.44 & 0.49 \\
\hline 19 & 0.80 & 1.14 & 0.54 & 0.59 & 0.85 & 0.44 & 0.52 \\
\hline 20 & 0.64 & 1.23 & 0.49 & 0.70 & 0.81 & 0.44 & 0.52 \\
\hline Mean $\pm S D$ & $0.82 \pm 0.15$ & $1.25 \pm 0.27$ & $0.63 \pm 0.20$ & $0.67 \pm 0.11$ & $0.91 \pm 0.27$ & $0.44 \pm 0.0022$ & $0.53 \pm 0.03$ \\
\hline \multicolumn{8}{|c|}{ Women } \\
\hline 1 & 1.26 & 1.31 & 0.63 & 0.89 & 1.12 & 0.54 & 0.64 \\
\hline 3 & 1.12 & 1.19 & 0.84 & 0.86 & NV & 0.54 & 0.65 \\
\hline 4 & 0.87 & 1.15 & 0.96 & 0.62 & NV & 0.54 & 0.63 \\
\hline 6 & 1.32 & 1.56 & 1.01 & 0.87 & 0.93 & 0.55 & 0.67 \\
\hline 9 & 0.99 & 1.65 & 1.11 & 0.86 & NV & 0.55 & 0.66 \\
\hline 10 & 1.04 & 1.09 & 1.05 & 0.84 & 1.44 & 0.54 & 0.69 \\
\hline 13 & 1.71 & 1.52 & 1.12 & 0.64 & 1.11 & 0.55 & 0.71 \\
\hline 16 & 1.15 & 1.33 & 0.83 & 0.55 & 0.98 & 0.54 & 0.65 \\
\hline Mean $\pm S D$ & $1.18 \pm 0.26$ & $1.35 \pm 0.21$ & $0.94 \pm 0.17$ & $0.77 \pm 0.14$ & $1.12 \pm 0.20$ & $0.54 \pm 0.0024$ & $0.66 \pm 0.03$ \\
\hline
\end{tabular}

windowing technique is applied (26). Rod windowing discards all detected coincidences that do not intersect the rod source (within a certain distance), and, as such, it reduces the influence of scatter, randoms, and emission spillover. To further validate quantitative accuracy, a comparison was made between blood-pool activity seen in the PET images and activity seen in manual samples.

The quantification of blood-pool activity in the left ventricle of the heart agreed well with the sampled blood activity, except for a few heavy-weight patients $(>100 \mathrm{~kg})$, suggesting that immuno-PET with ${ }^{89} \mathrm{Zr}$-cmAb U36 can be used to quantify the radiation dose of the whole body and normal organs of interest. However, as shown earlier with ${ }^{18}$ F-FDG PET, excessive body weight has negative effects on both quantitative and qualitative scan analysis (27-30). For ${ }^{89} \mathrm{Zr}$ studies, these effects may be even more pronounced because of the low positron emission abundance resulting in lower noise-equivalent counting rates than seen with ${ }^{18} \mathrm{~F}$-FDG studies. The low noise-equivalent counting rates could potentially hamper the accuracy and precision of the scatter correction, but further studies are required to fully understand the negative bias seen with heavier patients. Yet the association between patient weight and bias suggests that this effect is indeed related to scattercorrection issues. Despite this fact, a good agreement between PET and sample-derived blood-pool activities was seen for most other scans and subjects (Fig. 3). In future studies, with modern PET/CT scanners in combination with time-of-flight reconstruction and optimized scan protocols, it is probable that these problems will be of less magnitude, but this would require further validation as well.

Also in the assessment of tumor uptake, a good agreement was found between PET-derived data and data obtained from biopsies, despite the fact that there was a 1-d difference in assessment time (144 h after injection vs. $168 \mathrm{~h}$ after injection). This good agreement is quite remarkable because both methods are prone to errors. For example, for uptake assessment in tumor biopsies it is difficult to take a representative part of the tumor. For PET assessment, partial-volume effects will cause an underestimation of the tumor dose. This problem with partialvolume effects is a known limitation of PET, and correction methods are currently being developed to deal with this. At this point we can at least identify patients with low and high tumor uptake; therefore, it is possible to select the patients who may and may not benefit from therapy.

Although ${ }^{89} \mathrm{Zr}$ immuno-PET can become an important tool in the detection and treatment of cancer, there are some limitations to overcome. Drawing of the organ VOIs requires anatomic knowledge and training and is timeconsuming. However, with modern PET/CT scanners, ${ }^{89} \mathrm{Zr}$ immuno-PET data can be collected, along with a structural CT image, in 1 scanning session. The aligned CT data can then be used for a more accurate and automated VOI definition.

The mean radiation dose for patients in this study was about $40 \mathrm{mSv}$, which is high and will limit repeated application of ${ }^{89} \mathrm{Zr}$ immuno-PET. However, the introduction 
of the new-generation PET/CT scanners will also allow better-quality immuno-PET images to be obtained with a lower ${ }^{89} \mathrm{Zr}$ radioactivity dose. This can be concluded from preliminary PET/CT studies in which $37 \mathrm{MBq}$ of ${ }^{89} \mathrm{Zr}-$ trastuzumab was used for human epidermal growth factor receptor type 2 immuno-PET in breast cancer patients (15). Using ${ }^{89} \mathrm{Zr}-\mathrm{cmAb}$ U36 in combination with these scanners would mean an effective dose of about $20 \mathrm{mSv}$ for a whole-body scan. Theoretically, shorter-lived residualizing positron emitters such as ${ }^{64} \mathrm{Cu}\left(\mathrm{t}_{1 / 2}, 12.7 \mathrm{~h}\right)$ and ${ }^{86} \mathrm{Y}$ $\left(\mathrm{t}_{1 / 2}, 14.7 \mathrm{~h}\right)$ might give less dose exposure, although clinical experience with these positron emitters is limited. In the study by Cutler et al., immuno-PET with $370 \mathrm{MBq}$ of ${ }^{64} \mathrm{Cu}$-labeled intact $\mathrm{mAb} 1 \mathrm{~A} 3$ was evaluated for detection of colorectal cancer with a Siemens/CTI ECAT EXACT PET scanner, as was also used in the present study $(31,32)$. The average whole-body dose for these patients was 11.1 $\mathrm{mSv}$. However, because of the short half-life of ${ }^{64} \mathrm{Cu}$, most of the patients had to be imaged within $24 \mathrm{~h}$ after injection to obtain good-quality images. As shown in Figure 1A, for $\mathrm{cmAb}$ U36 the delineation of tumors is much better at later time points (72 and $144 \mathrm{~h}$ ), and, therefore, the use of ${ }^{64} \mathrm{Cu}$ might be a less suitable alternative. For the immuno-PET evaluation of antibody fragments, however, ${ }^{64} \mathrm{Cu}$ and ${ }^{86} \mathrm{Y}$ might be suitable candidates.

\section{CONCLUSION}

In the present study, we evaluated the safety, biodistribution, radiation dose, and potential for quantification of immuno-PET with ${ }^{89} \mathrm{Zr}$-labeled $\mathrm{cmAb}$ U36 in HNSCC patients. ${ }^{89} \mathrm{Zr}-\mathrm{cmAb}$ U36 was found to be safe and well tolerated in all subjects. Uptake of radioactivity in the heart, lungs, liver, kidneys, and spleen decreased over time, and increased uptake was seen only at tumor sites and in the thyroid of some of the patients. Quantitative analysis, comparing PET-derived blood-pool activity and sampled blood activity, showed good agreement, except for the patients with a body weight of more than $100 \mathrm{~kg}$. Also, a good agreement was found for the assessment of antibody uptake in tumors. The mean effective dose for the whole body was $0.53 \pm 0.03 \mathrm{mSv} / \mathrm{MBq}$ in men and $0.66 \pm 0.03$ $\mathrm{mSv} / \mathrm{MBq}$ in women.

\section{ACKNOWLEDGMENTS}

This study was supported by the Dutch Cancer Society, grant IKA VU2000-2155. This study was partly performed within the framework of the Center for Translational Molecular Medicine (CTMM; www.ctmm.nl) project AIRFORCE 03O-103.

\section{REFERENCES}

1. Oldham RK, Dillham RO. Monoclonal antibodies in cancer therapy: 25 years of progress. J Clin Oncol. 2008;26:1774-1777.

2. Börjesson PK, Jauw YW, Boellaard R, et al. Performance of immuno-PET with zirconium-89-labeled chimeric monoclonal antibody U36 in the detection of lymph node metastases in head and neck cancer patients. Clin Cancer Res. 2006;12:2133-2140.

3. Verel I, Visser GW, Van Dongen GA. The promise of immuno-PET in radioimmunotherapy. J Nucl Med. 2005;46(suppl):164S-171S.

4. Van Dongen GA, Visser GW, Lub-de Hooge MN, de Vries EG, Perk LR. Immuno-PET: a navigator in monoclonal antibody development and applications. Oncologist. 2007;12:1379-1389.

5. Wu AM. Antibodies and antimatter: the resurgence of immuno-PET. $J$ Nucl Med. 2009;50:2-5.

6. Nayak TK, Brechbiel MW. Radioimmunoimaging with longer-lived positronemitting radionuclides: potentials and challenges. Bioconjug Chem. 2009;20: 825-841.

7. Verel I, Visser GW, Boerman OC, et al. Long-lived positron emitters zirconium89 and iodine-124 for scouting of therapeutic radioimmunoconjugates with PET. Cancer Biother Radiopharm. 2003;18:655-661.

8. Perk LR, Visser GW, Vosjan MJ, et al. ${ }^{89} \mathrm{Zr}$ as a PET surrogate radioisotope for scouting biodistribution of the therapeutic radiometals ${ }^{90} \mathrm{Y}$ and ${ }^{177} \mathrm{Lu}$ in tumorbearing nude mice after coupling to the internalizing antibody cetuximab. $\mathrm{J} \mathrm{Nucl}$ Med. 2005;46:1898-1906.

9. Perk LR, Visser OJ, Stigter-Van Walsum M, et al. Preparation and evaluation of ${ }^{89} \mathrm{Zr}$-Zevalin for monitoring ${ }^{90} \mathrm{Y}$-Zevalin biodistribution with positron emission tomography. Eur J Nucl Med Mol Imaging. 2006;33:1337-1345.

10. Verel I, Visser GW, Boellaard R, Stigter-Van Walsum M, Snow GB, Van Dongen GA. Zirconium-89 immuno-PET: comprehensive procedures for the production of ${ }^{89} \mathrm{Zr}$-labeled monoclonal antibody. J Nucl Med. 2003;44:1271-1281.

11. Perk LR, Stigter-van Walsum M, Visser GW, et al. Quantitative PET imaging of Met-expressing human cancer xenografts with ${ }^{89} \mathrm{Zr}$-labelled monoclonal antibody DN30. Eur J Nucl Med Mol Imaging. 2008;35:1857-1867.

12. Brouwers AH, Verel I, Van Eerd JE, et al. PET radioimmunoscintigraphy of renal cell cancer using ${ }^{89} \mathrm{Zr}$-labeled G250 monoclonal antibody in nude rats. Cancer Biother Biopharm. 2004;19:155-163.

13. Nagengast WB, De Vries EG, Hospers GA, et al. In vivo VEGF imaging with radiolabeled bevacizumab in a human ovarian tumor xenograft. J Nucl Med. 2007;48:1313-1319.

14. Aerts HJLW, Dubois L, Perk LR, et al. Disparity between In Vivo EGFR expression and zirconium-89-labeled cetuximab uptake assessed by PET. $J$ Nucl Med. 2009;50:123-131.

15. Dijkers E, Lub-de Hooge MN, Kosterink JG, et al. Characterization of ${ }^{89} \mathrm{Zr}$ trastuzumab for clinical HER2 immunoPET imaging [abstract]. J Clin Oncol. 2007;25(June 20 suppl):3508.

16. De Bree R, Roos JC, Quak JJ, Den Hollander W, Snow GB, Van Dongen GA. Radioimmunoscintigraphy and biodistribution of technetium-99m-labeled monoclonal antibody U36 in patient with head and neck cancer. Clin Cancer Res. 1995;1:591-598.

17. Colnot DR, Quak JJ, Roos JC, et al. Phase I therapy study of rhenium-186labeled chimeric monoclonal antibody U36 in patients with squamous cell carcinoma of the head and neck. J Nucl Med. 2000;41:1999-2010.

18. De Bree R, Roos JC, Quak JJ, Den Hollander W, Snow GB, Van Dongen GA. Clinical screening of monoclonal antibodies 323/A3 (K931), cSF-25 (K984), and K928 for suitability of targeting tumors in the upper-aerodigestive and respiratory tract. Nucl Med Commun. 1994;15:613-627.

19. Heider KH, Mulder JW, Ostermann E, et al. Splice variants of the cell surface glycoprotein CD44 associated with metastatic tumour cells are expressed in normal tissues of humans and cynomolgus monkeys. Eur J Cancer. 1995;31A: 2385-2391.

20. Prince ME, Sivanandan R, Kaczorowski A, et al. Identification of a subpopulation of cells with cancer stem cell properties in head and neck squamous cell carcinoma. Proc Natl Acad Sci USA. 2007;104:973-978.

21. Mack B, Gires O. CD44s and CD44v6 expression in head and neck epithelia. PLoS One. 2008;3:e3360.

22. Boellaard R, Hoekstra OS, Lammertsma AA. Software tools for standardized analysis of FDG whole body studies in multi-center trials [abstract]. J Nucl Med. 2008;49(suppl 1):159P.

23. Stabin MG, Sparks RB, Crowe E. OLINDA/EXM: the second-generation personal computer software for internal dose assessment in nuclear medicine. J Nucl Med. 2005;46:1023-1027.

24. Sgouros G. Bone marrow dosimetry for radioimmunotherapy: theoretical considerations. J Nucl Med. 1993;34:689-694.

25. Plaizier MA, Roos JC, Teule GJ, et al. Comparison of non-invasive approaches to red marrow dosimetry for radiolabelled monoclonal antibodies. Eur J Nucl Med. 1994;21:216-222.

26. Jones WF, Digby WM, Luk WK, Casey ME, Byars LG. Optimizing rod window width in positron emission tomography. IEEE Trans Med Imaging. 1995;14: 266-270. 
27. Tatsumi M, Clark PA, Nakamoto Y, Wahl RL. Impact of body habitus on quantitative and qualitative image quality in whole-body FDG-PET. Eur J Nucl Med Mol Imaging. 2003;30:40-45.

28. Botkin CD, Osman MM. Prevalence, challenges, and solutions for ${ }^{18} \mathrm{~F}-\mathrm{FDG}-\mathrm{PET}$ studies of obese patients: a technologist's perspective. J Nucl Med Technol. 2007; 35:80-83.

29. Halpern BS, Dahlbom M, Quon A, et al. Impact of patient weight and emission scan duration on PET/CT image quality and lesion detectability. $\mathrm{J}$ Nucl Med. 2004;45:797-801.
30. Halpern BS, Dahlbom M, Auerbach MA, et al. Optimizing imaging protocols for overweight and obese patients: a lutetium orthosilicate PET/CT study. J Nucl Med. 2005;46:603-607.

31. Philpott GW, Schwarz SW, Anderson CJ, et al. RadioimmunoPET: detection of colorectal carcinoma with positron-emitting copper-64-labeled monoclonal antibody. J Nucl Med. 1995;36:1818-1824.

32. Cutler PD, Schwarz SW, Anderson CJ, et al. Dosimetry of copper-64-labeled monoclonal antibody $1 \mathrm{~A} 3$ as determined by PET imaging of the torso. $\mathrm{J} \mathrm{Nucl}$ Med. 1995;36:2363-2371. 